\title{
N9 $0-22951$
}

\section{SPATIAL DISPLAYS AS A MEANS TO INCREASE PILOT SITUATIONAL AWARENESS}

\author{
Delmar M. Fadden, Dr. Rolf Braune, and John Wiedemann \\ Boeing Commercial Airplane Company \\ Seattle, Washington
}

At least three elements influence the performance of an operator who must make a system achieve a desired goal: (1) the dynamics of the system itself, (2) the nature of the possible inputs, and (3) the means whereby the operator views the information concerning the desired and actual state of the system (e.g., Poulton, 1974; Wickens, 1984; and Wickens, 1987). In conventional airplanes manual control involves the coordination of "inner loop" controls. In this task the pilot is responsible for continuous manipulation of the controls to compensate for disturbances. Primary displays (fig. 1) provide the several essential flight parameters which the pilot is required to monitor, interpret, transform, and integrate.

It has long been recognized that intense concentration is necessary for a pilot to achieve high tracking performance using only "raw data." The underlying need for such concentration stems from the effort necessary to obtain timely error, error rate, and control input information in each of the three flight axes. Precision instrument approaches often have higher minimums if a suitable flight director or autopilot is not available and in use. Most pilots have come to depend on these aids. Some pilots express doubt about the precision of their own tracking ability any time they are unavailable.

Flight directors, which came into widespread airline use in the 1960 s, aid the pilot in achieving improved performance by combining the error and error rate information; producing a control command appropriate to the situation. This command is then compared with the existing control input and the difference displayed as a steering command. The generation of the steering command entails automation of several logical and mathematical operations. Of course the pilot must set up the proper task for the flight director to perform and must follow the steering commands. In typical applications the automation is sufficiently complete that the pilot has no required intermediate data-interpretation role beyond that of recognizing and following the steering command.

While use of the flight director improves performance in precision tasks, it does not significantly reduce the continuous attention demands imposed on the pilot. Use of a path-following autopilot mode automates the process one step further by coupling the steering command to the control surfaces. Relieved of the continuous steering requirement, the pilot is able to devote more time to other tasks.

Both flight directors and autopilots achieve impressive performance gains. A side effect of these gains is a reduction in the necessity for the pilot to maintain a high level of awareness of the elements pertinent to the control task; namely the path error, error rate, and control input. To be sure, all modern aircraft present these parameters and most airline operating procedures dictate that the pilot monitor them while using either the flight director or autopilot. However, the monitoring task is fundamentally different from that of developing a control input given only "raw" data. In particular, the dynamic decision-making demands of the monitoring task are much lower than those of the control task. 
Spatial displays, together with enhanced manual control, offer an opportunity to achieve the same high performance achieved with autopilots and flight directors while improving the pilot's overall situational awareness, particularly during flight tasks other than final approach. This is accomplished by revising the split of responsibilities between the pilot and the aircraft automation.

In transport operations, the need to alter the velocity (flightpath angle, track angle, or speed) is much less frequent than the need to compensate for wind effects, turbulence, configuration changes, and speed changes. In terminal area operations, the number of required velocity changes may be an order of magnitude or more lower than those attitude changes necessary to maintain a velocity. Furthermore, the needed velocity changes are typically separated by many seconds. By assigning the velocity-hold task to the basic flight-control system, the majority of the attitude adjustments can be made transparent to the pilot. This type of control frees the pilot from the continuous attention requirement of attitude steering while maintaining the pilot's direct involvement in airplane guidance.

Spatial displays make it possible for the pilot to be directly involved in developing the path error information and in selecting the specific tactic to be employed in correcting the error. To make this practical, current position and velocity information must be displayed in a consistent context. Operational displays based on work done at Boeing, NASA Langley, RAE-Weybridge, and other places have shown that a map display, with track angle and speed shown by means of predicted future positions, provides a suitable context.

The first generation of commercial airline spatial displays are in operation on the Boeing 757 and 767 and the Airbus A-310. These displays take the form of CRT maps with various types of integral predictors (fig. 2). The format consistency of these displays is quite high and pilot acceptance has been exceptionally good. The CRT maps are used for planning and assessing all types of lateral maneuvers. Direct manual aircraft control is still accomplished by reference to a separate attitude instrument, but virtually all of the decisions to maneuver laterally can be made looking at information contained in the map display.

The success of the map display and the potential for flightpath angle and track angle control to be used on the next generation of commercial aircraft encouraged us to consider expanding the role of spatial displays. Data from the NASA Aviation Safety Reporting System identifies altituderelated errors as the single largest category of reported problems (Reynard, Ames Research Center, 1987 , personal communication). While the immediate causes of the reported errors are quite varied, we see a common thread emerging. The pilot's awareness of the vertical flight situation in most instances does not match the reality of the flight plan, the ATC clearance, or the equipment setup. A spatial display should be an ideal means of improving the pilot's vertical situation aware-
ness (Baty, 1976).

For most transport flight operations except takeoff and landing, the tracking accuracy required of the pilot is at least an order of magnitude higher for the vertical task than for the lateral task. Typical tracking-performance goals as perceived by the pilot away from final approach are $\pm 50 \mathrm{ft}$ of altitude and $\pm 0.5^{\circ}$ of a VOR radial. At $40 \mathrm{n}$. mi. from the VOR station, $\pm 0.5^{\circ}$ corresponds to over $\pm 2000 \mathrm{ft}$. At this point the accuracy ratio is $40: 1$. Even on final approach the vertical accuracy requirements exceed the lateral by at least $2: 1$. If a conformal 3-D display were used with sufficient resolution to satisfy the vertical task, the pilot would be overworked laterally. This concern, along with the difficulty of presenting future trend information in a forward-looking 
display, lead us to concentrate on a separate 2-D side-view display for the majority of vertical situation information (Grunwald, 1980; and Filarsky, 1983).

Some past aircraft programs have referred to the attitude display as a vertical situation display. We prefer to use the more conventional terminology, ADI (attitude director indicator) or PFD (primary flight display) for the forward-looking display of attitude information and other fundamental flight data. We refer to a side-looking or profile display as a vertical-situation display and expect that the pilot would obtain the majority of overall vertical situation awareness from this display (fig. 3).

Over the past 2 yr we have been exploring ways of developing a useful and effective means to portray vertical-situation information. There are a number of practical problems which narrow the possible format options for vertical flight information. The remainder of this paper will outline the larger hurdles and indicate what progress has been made in solving them.

Three issues appear to be fundamental to the development of a successful vertical situation display:

1. Handling of the large difference in resolution requirements between the longitudinal and vertical flight tasks.

2. Determination of the appropriate level of control information to be contained in the instrument.

3. Selection of a display context which will be intuitive to the pilot and provide useful assistance for on- and off-path vertical maneuvering.

\section{SCALING ISSUES}

The disparity which exists between vertical and lateral resolution requirements applies as well to vertical and longitudinal information. In fact, since time constraints are seldom tighter than a minute or more, the difference in resolution requirements can be well in excess of two orders of magnitude. With this large a difference, equal vertical and horizontal display scaling is clearly impractical. By using a flightpath predictor we have been able to achieve a balance between vertical tracking performance and the desired path preview capability.

Initial test results indicate that when the vertical situation is presented spatially, a steady increase in mean deviation from an optimal descent occurs as scale resolution is decreased (fig. 4). However, even the largest deviation is significantly less than the lowest mean without the spatial graphics. This result could be attributed to the difference in the tactics the subject pilots employed to accomplish the task under the two presentations. Without the graphics the pilots had to mentally integrate various analog quantities according to their own individual rules of thumb. As can be seen in figure 5, this results in an overall greater deviation from the optimal descent strategy and more variance among the individual pilot deviations. When given a spatial presentation of the situation, the subject pilots employed similar path-following tactics, resulting in greater tracking precision and a lower-rated workload level. 
The fact of unequal scales causes the angle representations on the display to be exaggerated vertically. Through a preliminary test series we found that scale differences of as much as $20: 1$ do not have a negative influence on typical airline flying tasks. Obviously aircraft with significantly greater climb or descent capabilities than transports would encounter difficulty at lower-scale ratios. What appears more important to the pilots is that the longitudinal scaling of the side-view display and the map display be congruent so that the rate of movement between the two is compatible.

Another result from our initial investigations reveals that a digital readout of altitude takes on added importance as scale resolution is decreased (fig. 6). In seeking the proper balance between scale resolution for precision and scale range for preview, it was shown that a digital readout of altitude provides a good vernier indication while the graphics provides the necessary "big picture" overview. The graphic spatial information is effective in drawing the pilot's attention to the digital readout when precise control is needed.

\section{CONTROL ISSUES}

In all of today's transport aircraft, manual control is exercised using the attitude display with follow-up reference to the situational displays. This is the case for map-display-equipped aircraft as well. Laterally the track angle is two integrations removed from aircraft roll rate, over which the pilot has direct control. The resulting time delay between control input and map response is too long for track angle to provide primary inner-loop feedback to the pilot. Even when lateral acceleration is used to create a prediction of the dynamic path which will be flown, the pilot's primary control feedback comes from the bank indication on the attitude indicator.

Vertically the conventional control parameter is pitch rate. This term is separated from flightpath angle by a single integration and some higher-order dynamics. For transports this places the flightpath response on the order of 1-2 sec behind the control input; long enough to be useless as the primary feedback term for most situations and short enough to interact negatively with pitch feedback. The primary dynamic term in the vertical situation is flightpath angle. Furthermore, flightpath angle, rather than pitch attitude, can be readily assessed in terms of the geometry or energy conditions of the vertical situation. If the response dynamics of flightpath angle were not so close to that of pitch attitude, the separation of control and situation assessment, which works very well in the lateral case, could be established for the vertical case as well.

Beginning with experimental work on the Boeing SST in the late 1960s and continuing through the early phases of the NASA TCV program, we became convinced that if flightpath angle, along with suitable situational reference information, is available to the flight crew, the crew will attempt to use it for control. Without good matching of the control and display dynamics, pilot
workload may well increase.

If a flightpath-angle command-control system is in use, it is possible to display the flightpath which will be held. This term can be made as responsive as necessary to support the pilot's need for timely information. If a more conventional control system is used, a filter with appropriate lead compensation can be added to quicken the dynamics of the flightpath angle information (Bray,
1981). 
The key situational element which makes control possible is the flightpath prediction based on flightpath angle. Remove the prediction and control reverts to conventional techniques. However, without the prediction, the usefulness of the display for enhancing current situational awareness is dramatically reduced. Even maintaining a constant altitude is difficult without the prediction. Thus the question about the desired level of control information is not an independent issue. If the display is to be useful, it must contain dynamic flightpath information. The presence of such information means that the display will be used for control. The real issue, then, is how to match the control and display dynamics to the information-processing capabilities of the pilot.

\section{DISPLAY CONTEXT}

The third fundamental issue has to do with matching the frame of reference of the display to that of the pilot. The vertical component is straightforward. However, the options for the horizontal component are more complex. If information concerning the planned route of flight were always available and current, then distance along the route would be a good choice. However, the planned route is not always available. Furthermore, one of the more important uses of the display is during operations when the airplane is intentionally away from the planned path.

For these situations a narrow slice ahead of the airplane would be more useful. In either case close coordination between the vertical and horizontal situation displays is essential.

Development work aimed at clarifying the format orientation issue is now under way. We expect to have an understanding of the major tradeoffs late this year.

\section{CONCLUSIONS}

Our experience raises a number of concerns for future spatial-display developers. While the promise of spatial displays is great, the cost of their development will be correspondingly large. The cost goes well beyond time and materials. The knowledge and skills which must be coordinated to ensure successful results is unprecedented. From the viewpoint of the designer, basic knowledge of how human beings perceive and process complex displays appears fragmented and largely unquantified. Methodologies for display development require prototyping and testing with subject pilots for even small changes. Useful characterizations of the range of differences between individual users is nonexistent or at best poorly understood. The nature, significance, and frequency of interpretation errors associated with complex integrated displays is unexplored and undocumented territory.

Graphic displays have intuitive appeal and can achieve face validity much more readily than earlier symbolic displays. The risk of misleading the pilot is correspondingly greater. Thus while we in the research community are developing the tools and techniques necessary for effective spatial-display development, we must educate potential users about the issues so they can make informed choices. The scope of the task facing all of us is great. The task is challenging and the potential for meaningful contributions at all levels is high indeed. 


\section{REFERENCES}

Baty, D. L. (1976) Rationale and Description of a Coordinated Cockpit Display for Aircraft Flight Management, NASA Technical Memorandum 3457, November 1976, Ames Research Center, Moffett Field, CA.

Bray, R. S.; and Scott, B. C. (1981) A Head-Up Display for Low Visibility Approach and Landing. Proceedings AIAA 19th Aerospace Sciences Meeting, January 12-15, St. Louis, MO.

Filarsky, S. M.; and Hover, G. W. (1983) The Command Flight Path Display. Proceedings of the Society for Information Displays, pp. 196-197.

Grunwald, A. J.; Robertson, J. B.; and Hatfield, J. J. (1980) Evaluation of a Computer Generated Perspective Tunnel Display for Flight Path Following. NASA Langley Tech Report \#1736.

Poulton, E. C. (1974) Tracking Skills and Manual Control. New York: Academic Press.

Wickens, C. D. (1984) Engineering Psychology and Human Performance. Columbus, OH: Charles Merrill.

Wickens, C. D. (1987) The Effects of Control Dynamics on Performance. In K. Boff and L. Kaufman (Eds.) Handbook of Perception and Performance. Dayton, OH: University of Dayton Research Institute. 


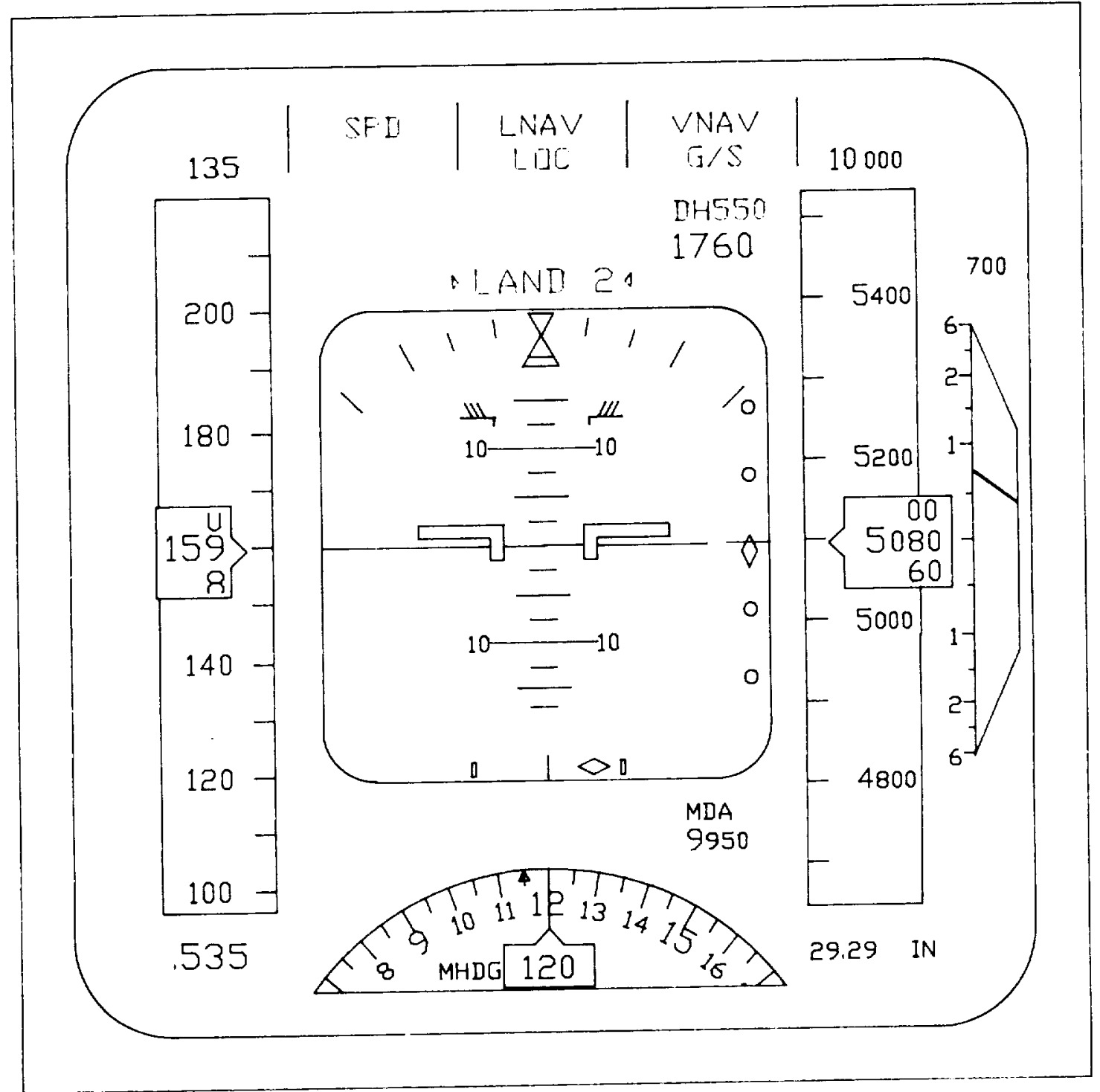

Figure 1.- Primary flight display. 


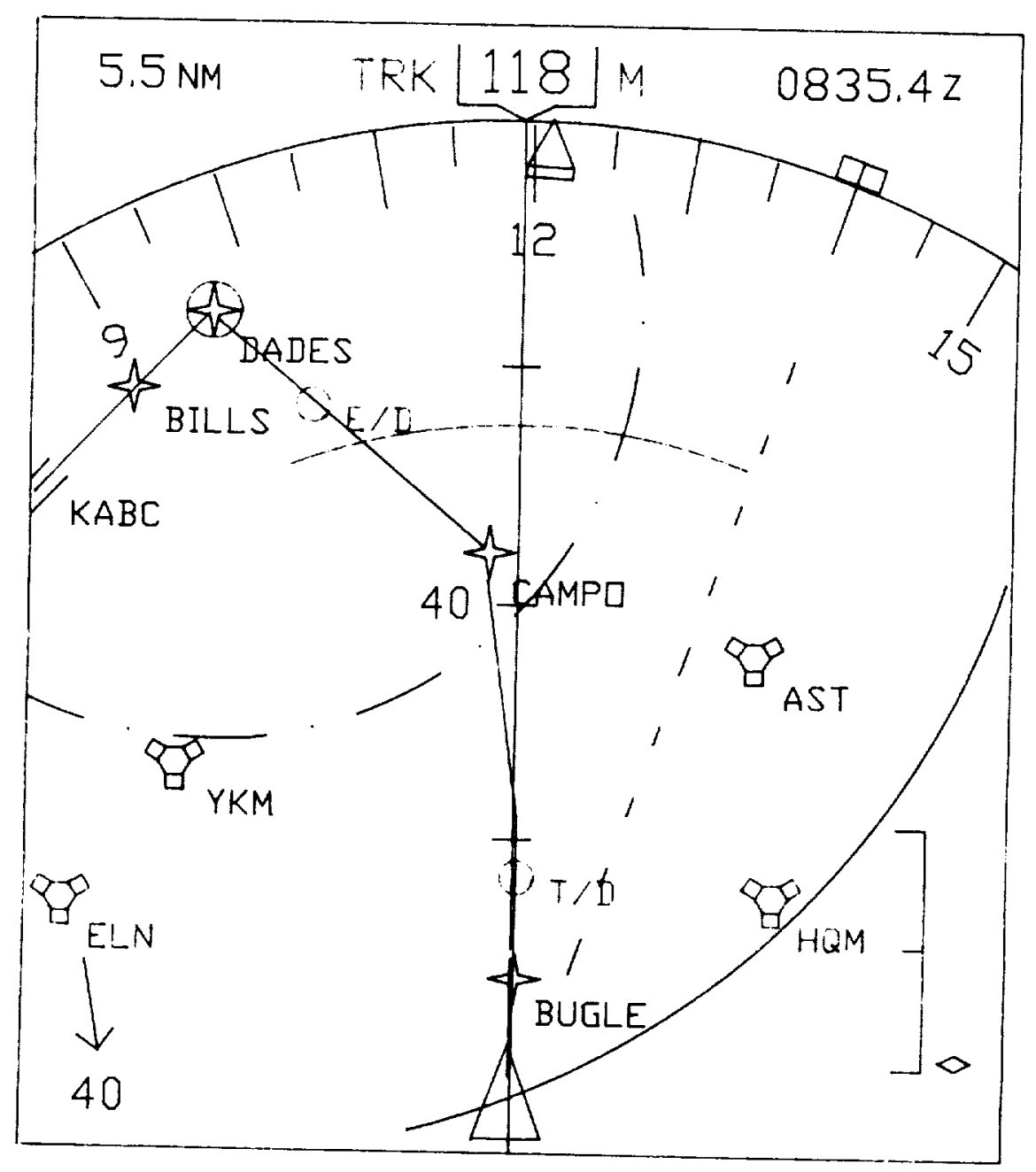

Figure 2.- Map display. 


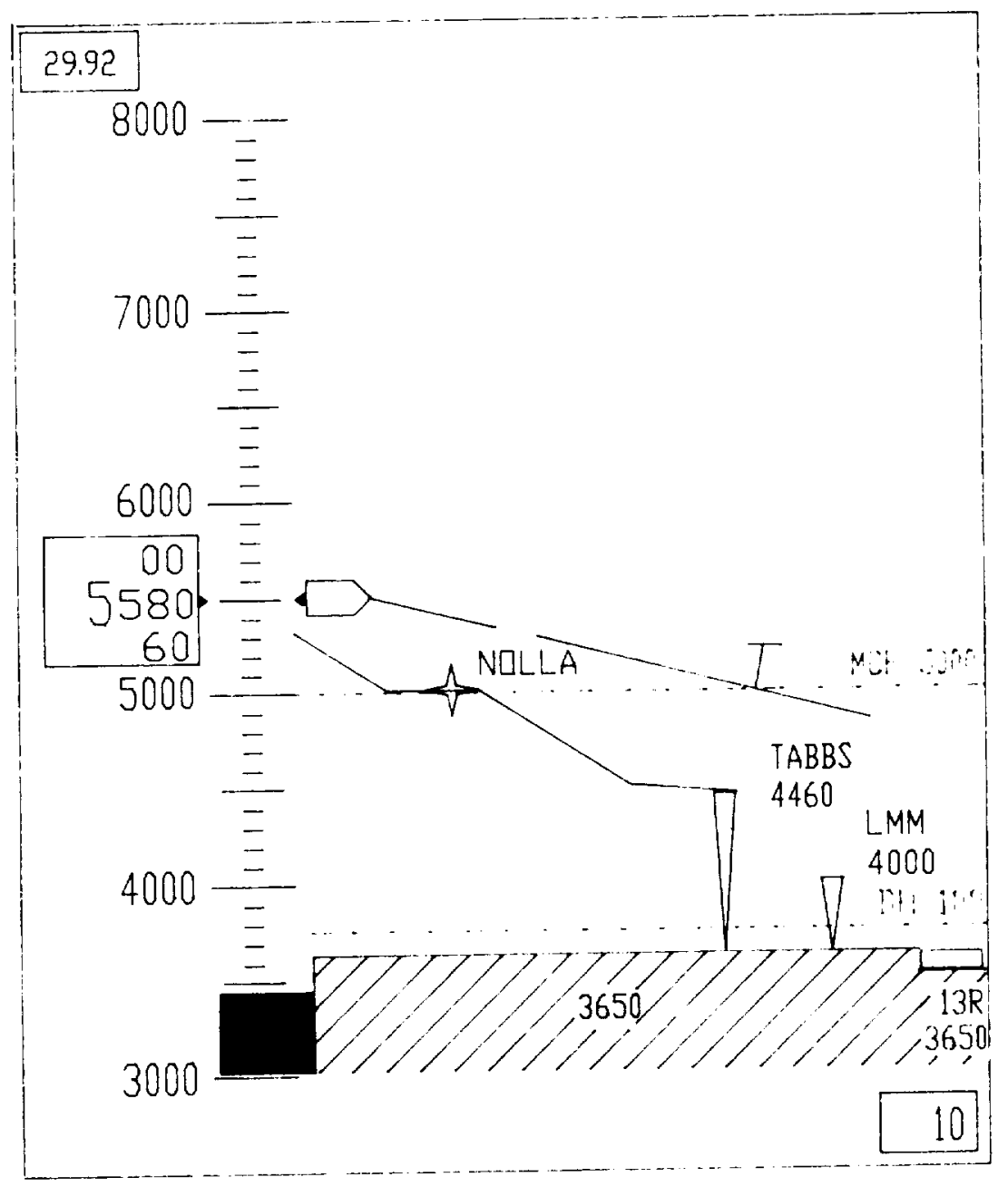

Figure 3.- Vertical situation display. 


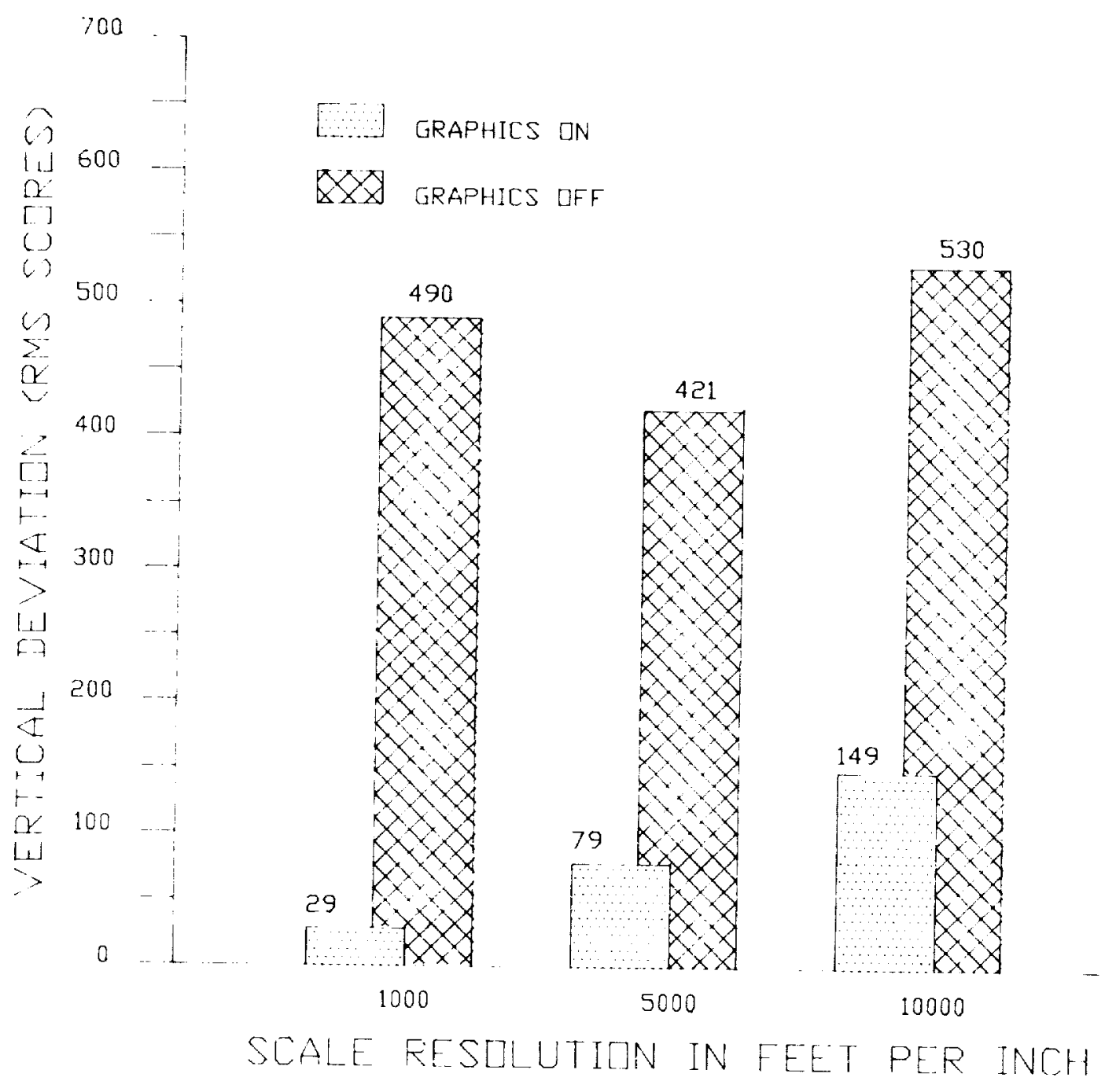

Figure 4.- Overall means for graphics $\times$ resolution. 
ORIGINAL PREE RE OF POOR QUAEIT

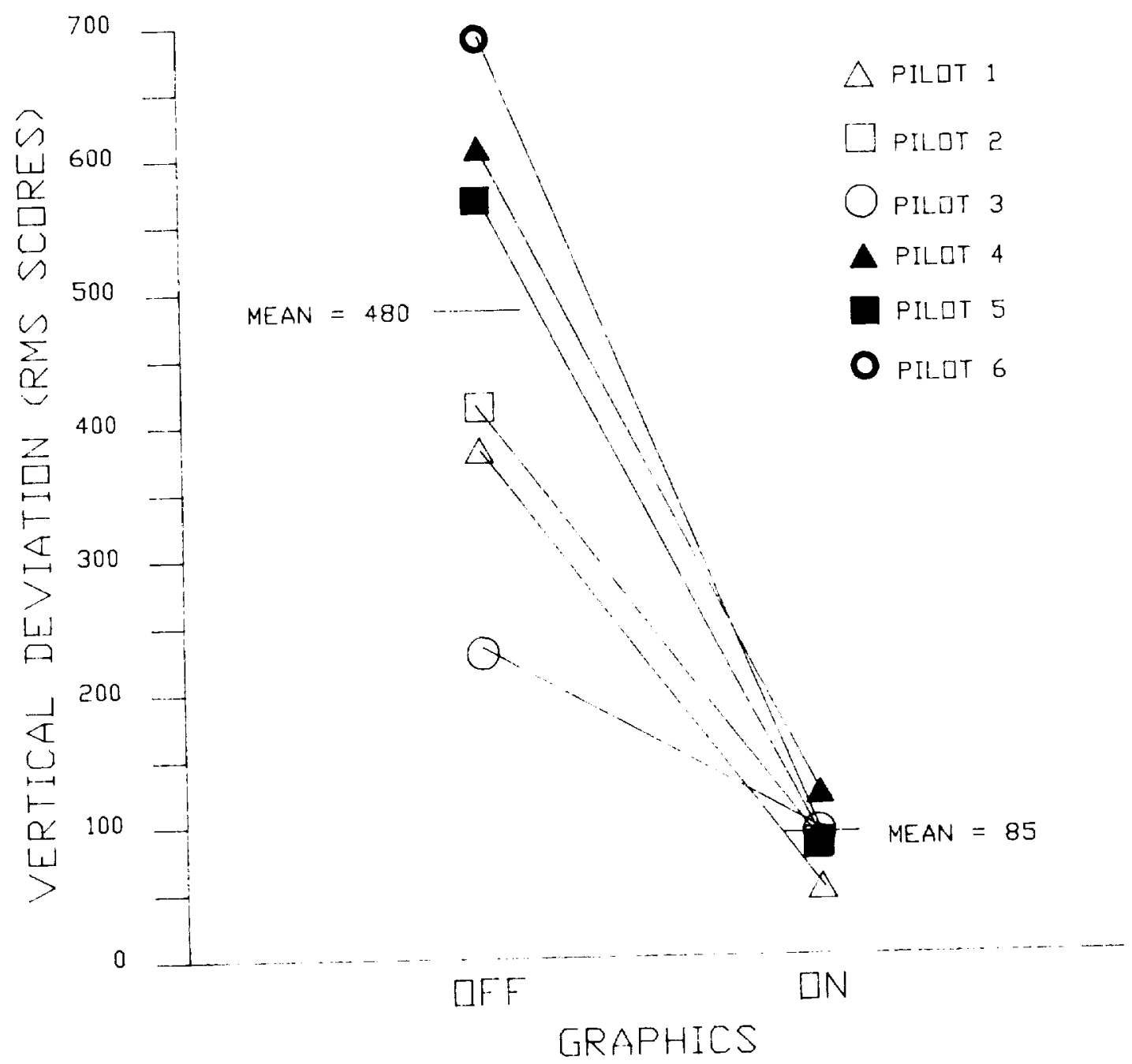

Figure 5.- Overall means for graphics $\times$ pilots. 


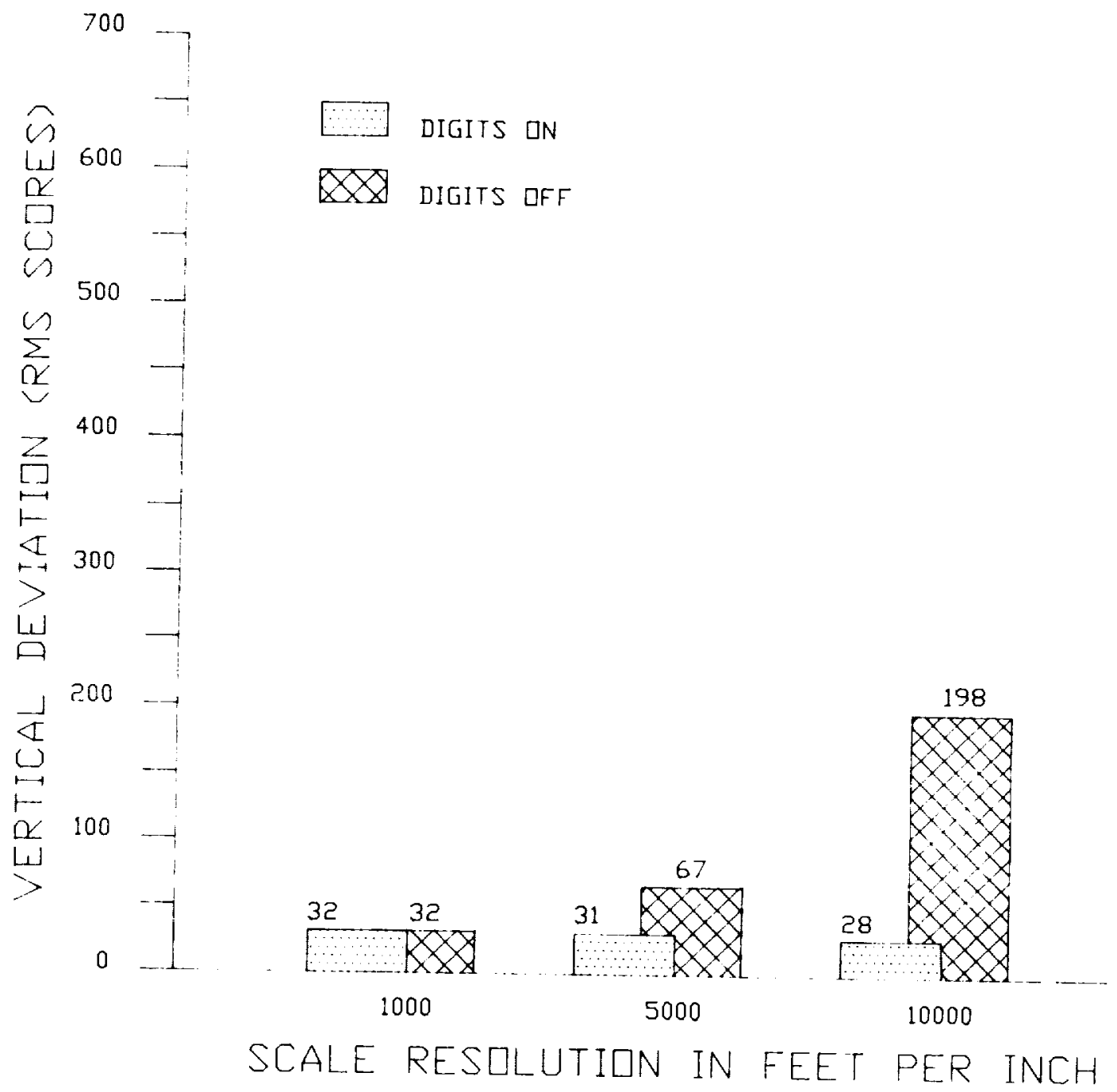

Figure 6.- Level-off segment (graphics on) means for digits $\times$ resolution. 